Supporting Information for

\title{
Antibacterial and Antifouling Hybrid Ionic-Covalent Hydrogels with Tunable Mechanical Properties
}

Jing Zhang*, Biao Shen, Lingdong Chen, Liqun Chen, Jiaying Mo, Jie Feng*

College of Materials Science \& Engineering, Zhejiang University of Technology,

Hangzhou, Zhejiang 310014, China

*corresponding authors:

E-mail addresses: zhangjing@zjut.edu.cn (J. Zhang), fengjie@zjut.edu.cn(J. Feng). 

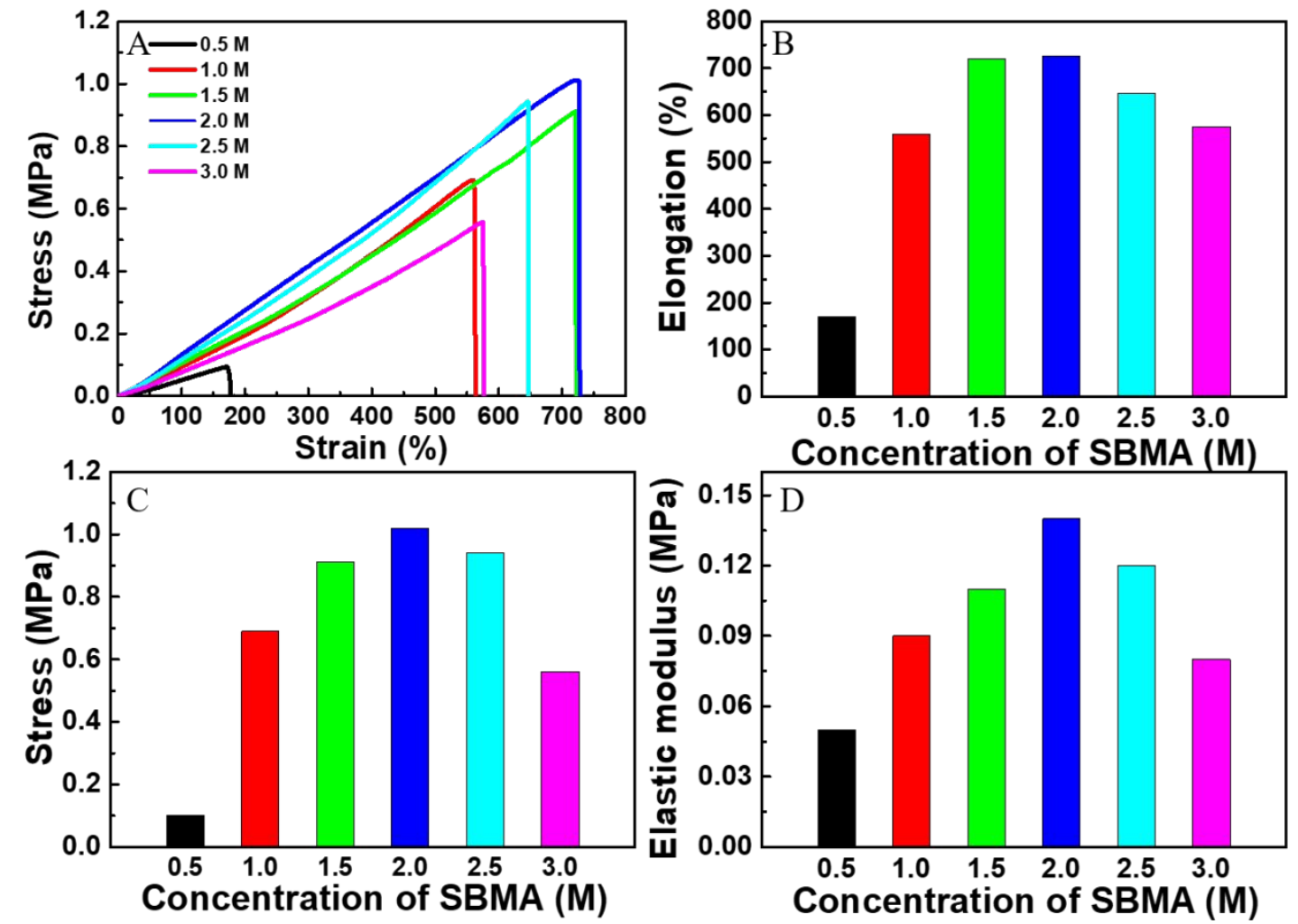

Figure S1. (A) Tensile curves, (B) elongation, (C) fracture stress, (D) elastic modulus of the $\mathrm{CZ}-\mathrm{Cit}^{3-}$ hydrogels with different concentrations of SBMA.
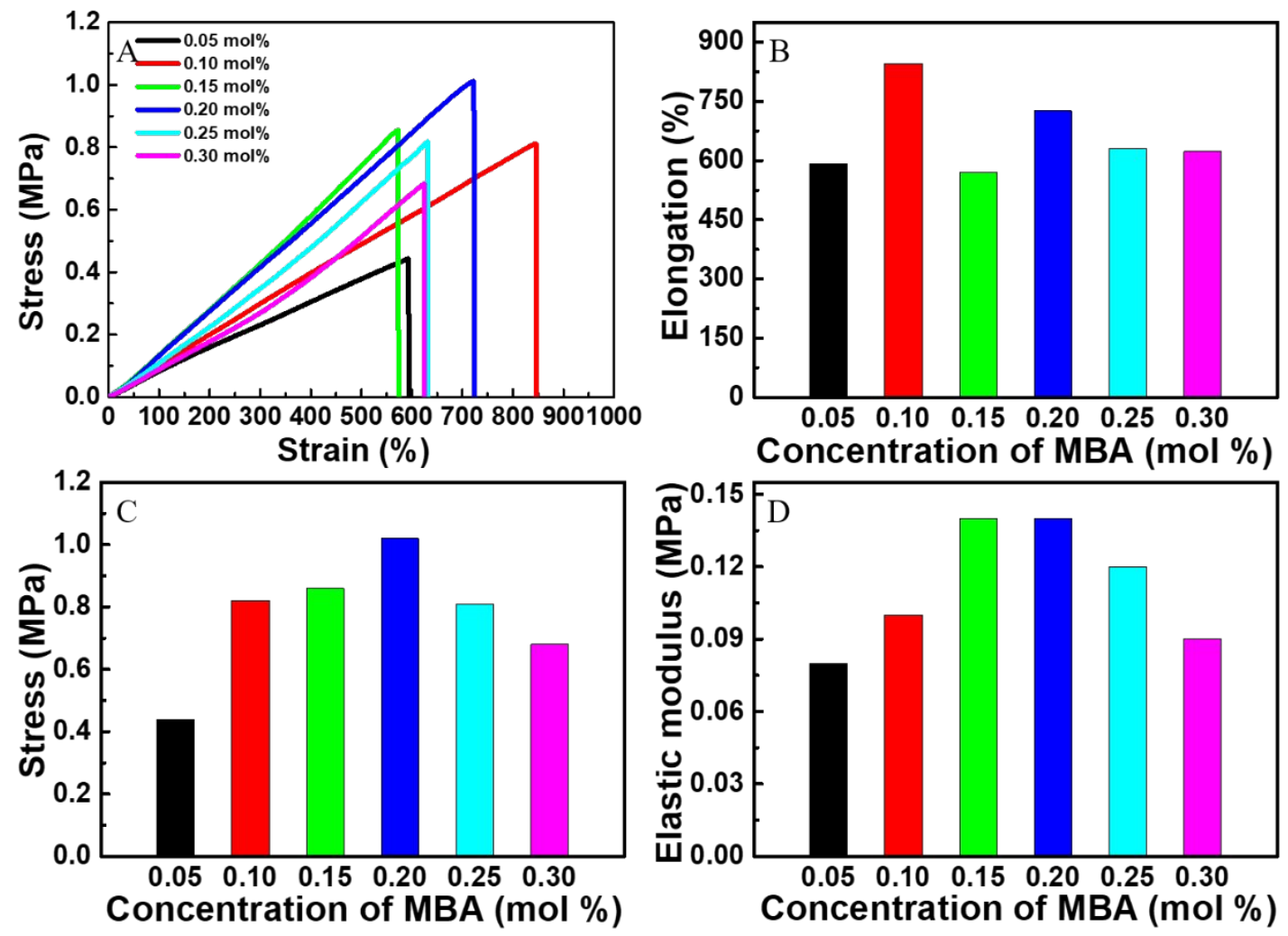

Figure S2. (A) Tensile curves, (B) elongation, (C) fracture stress, (D) elastic modulus of the

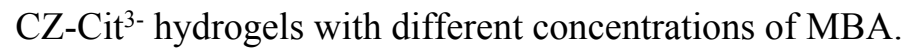



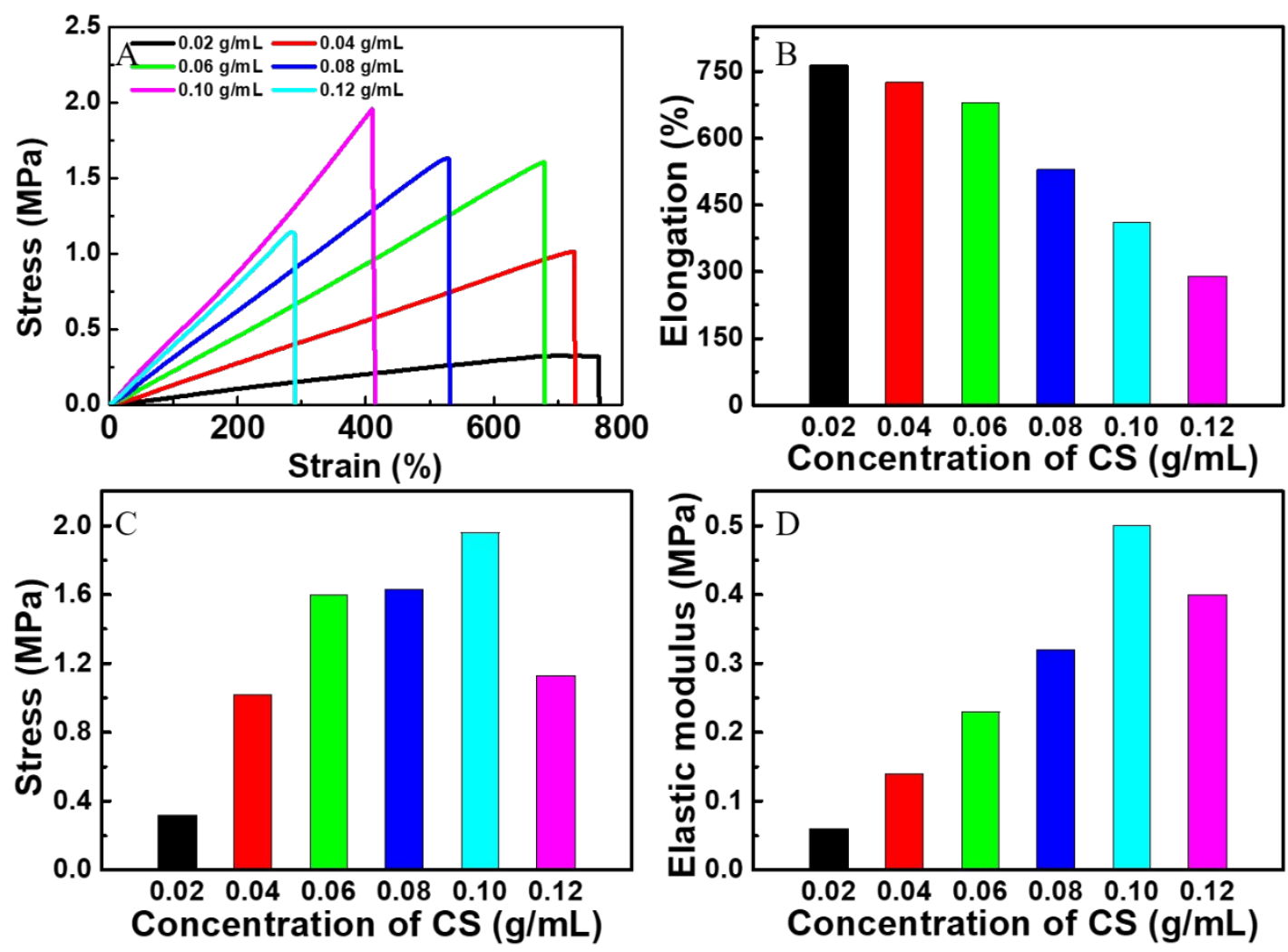

Figure S3. (A) Tensile curves, (B) elongation, (C) fracture stress, (D) elastic modulus of the CZ-Cit- hydrogels with different concentrations of CS.
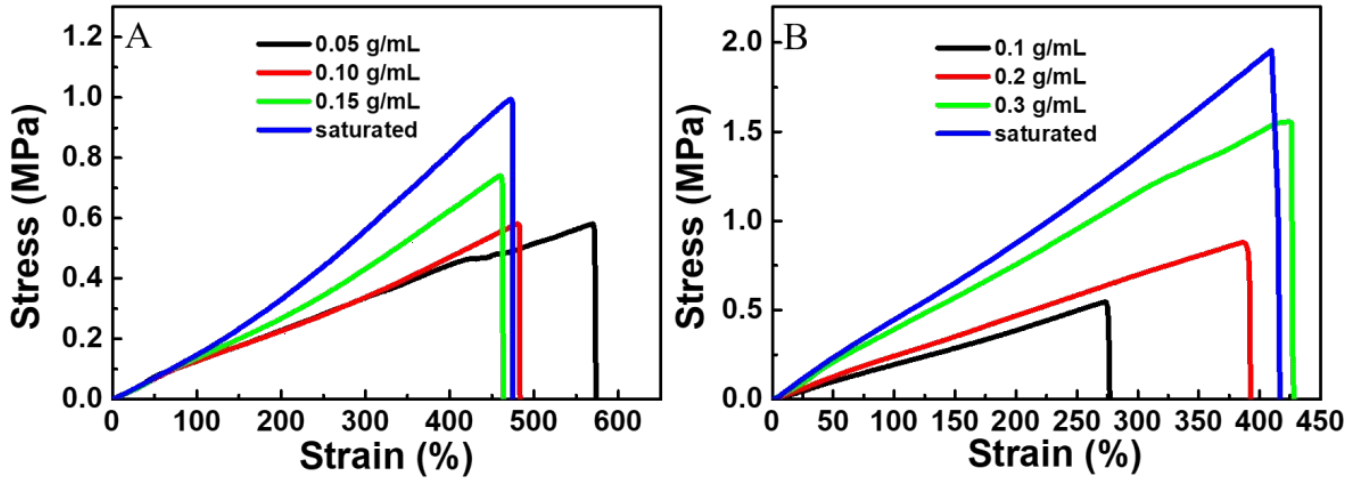

Figure S4. Tensile curves of the $\mathrm{CZ}_{-}-\mathrm{SO}_{4}{ }^{2-}(\mathrm{A})$ and $\mathrm{CZ}_{-} \mathrm{Cit}^{3-}$ (B) hydrogels immersed in different concentrations of salt solution. 

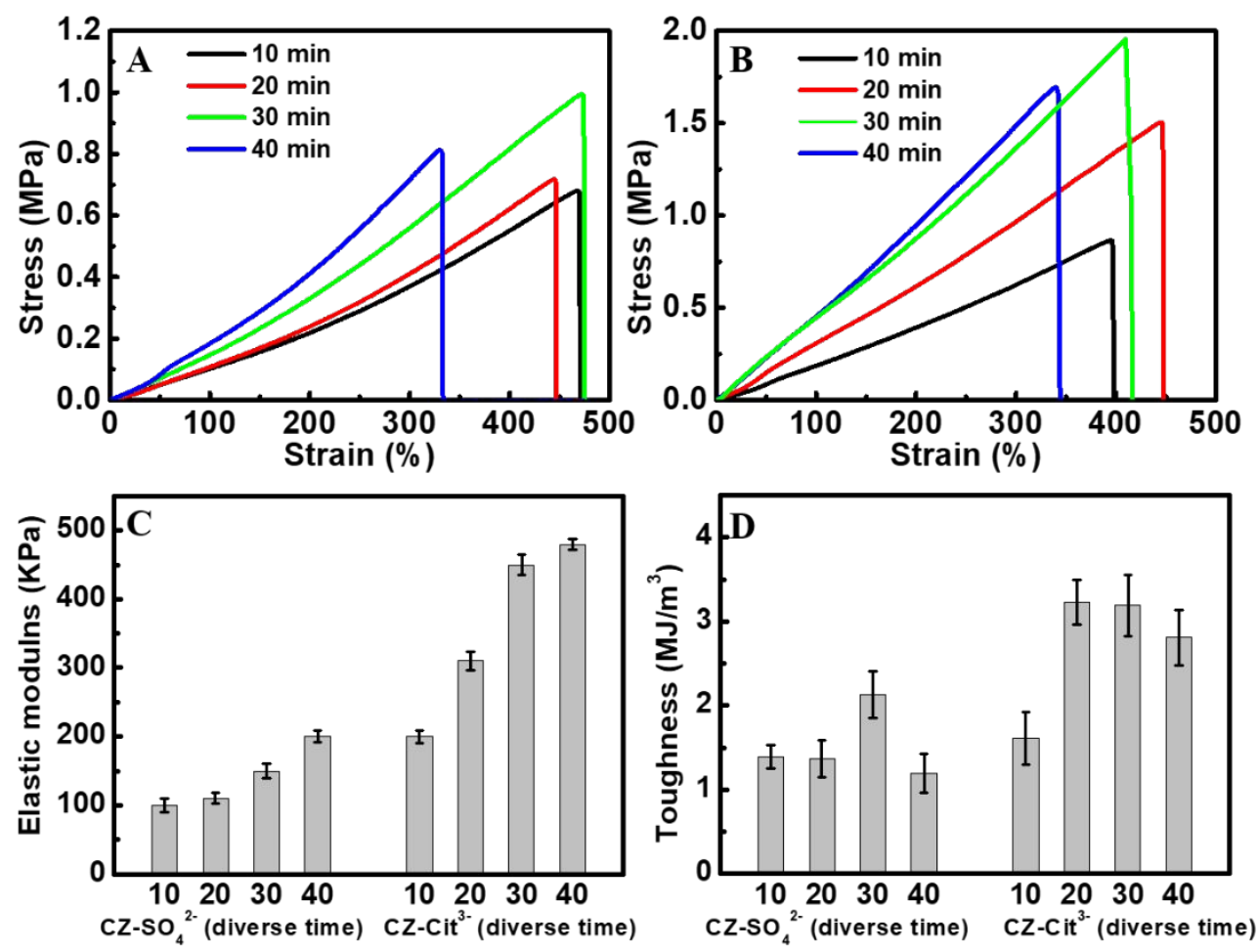

Figure S5. Tensile curves of the $\mathrm{CZ}-\mathrm{SO}_{4}{ }^{2-}(\mathrm{A})$ and $\mathrm{CZ}-\mathrm{Cit}^{3-}(\mathrm{B})$ hydrogel, $(\mathrm{C})$ elastic modulus, (D) toughness of DN hydrogels versus post-crosslinking times (10, 20, 30, $40 \mathrm{~min})$.
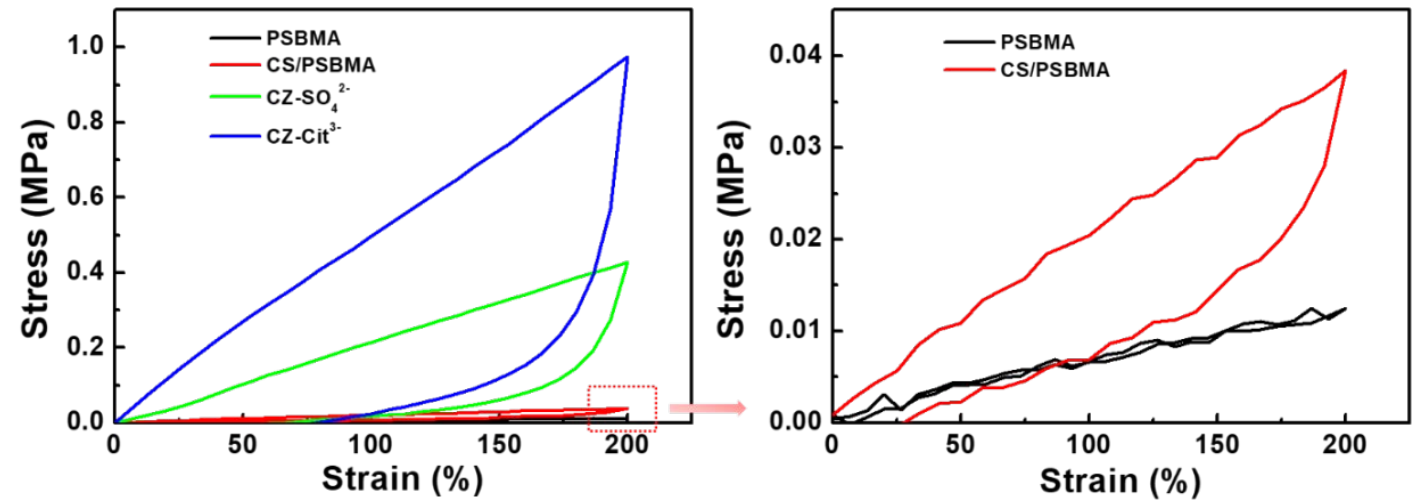

Figure S6. The loading-unloading curves of the PSBMA SN hydrogel, CS/PSBMA composite hydrogel, $\mathrm{CZ}-\mathrm{SO}_{4}{ }^{2-} \mathrm{DN}$ hydrogel and $\mathrm{CZ}-\mathrm{Cit}^{3-} \mathrm{DN}$ hydrogel at strain of $200 \%$.
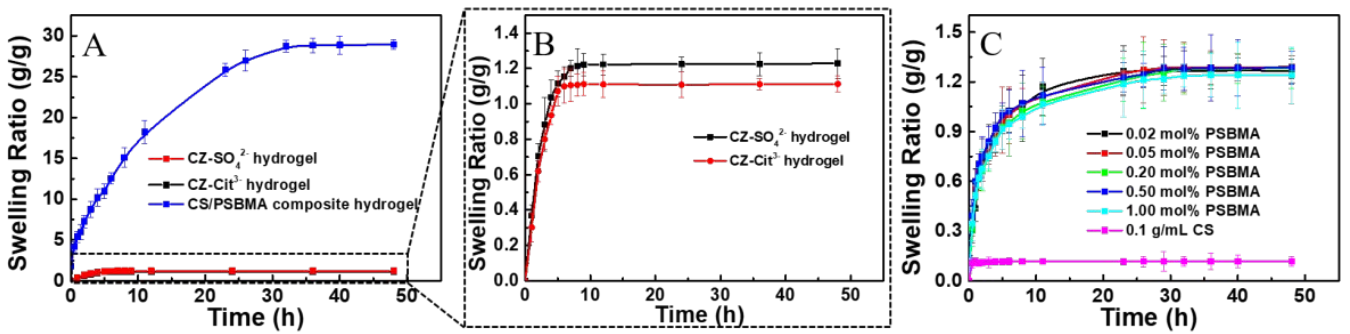

Figure S7. Swelling ratio (g/g) of (A) CS/PSBMA composite hydrogel and CS/PSBMA DN hydrogel and (C) PSBMA hydrogels and CS SN hydrogel. (B) is a partial enlargement of (A). 


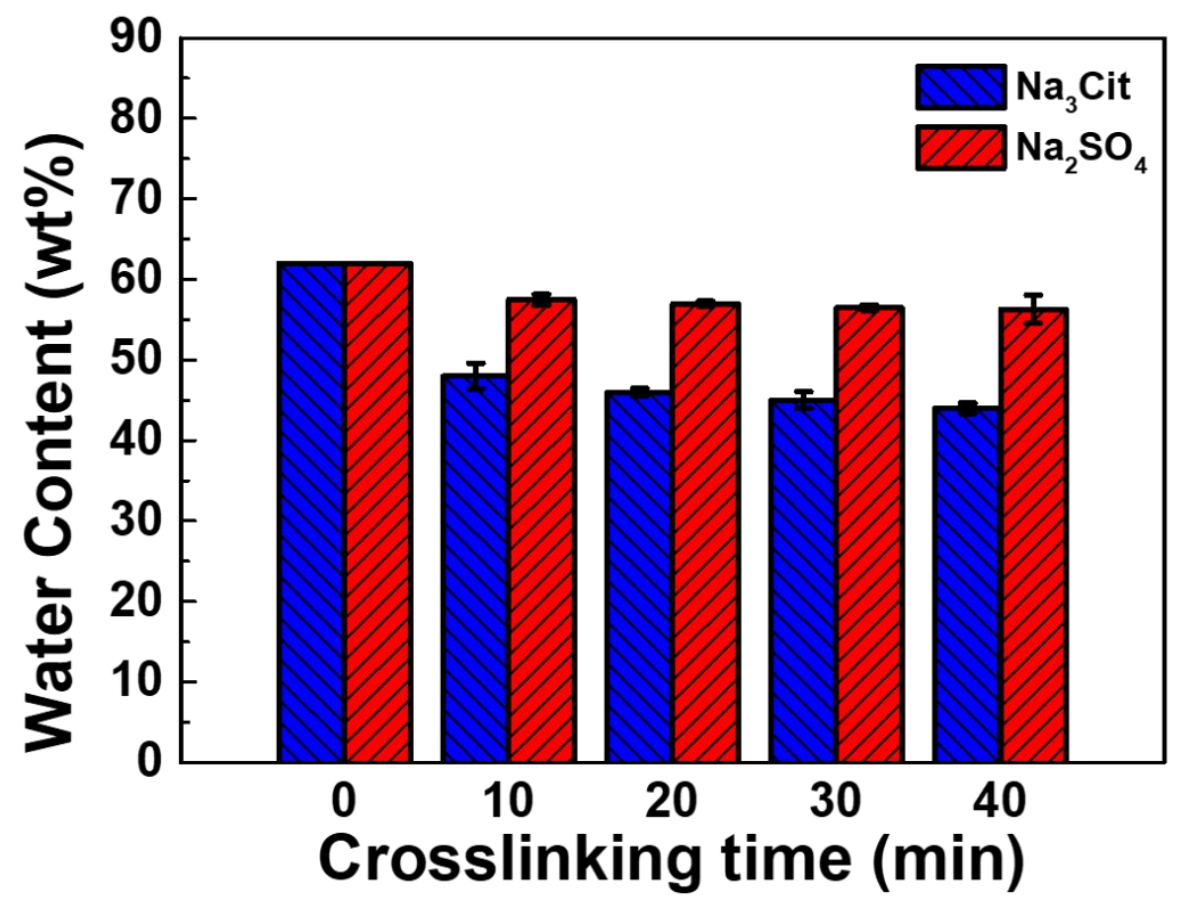

Figure S8. Water contents percentage of DN hydrogels versus post-crosslinking times. 